\title{
Structural, magnetic, and transport properties of the giant magnetoresistive perovskites $\mathrm{La}_{2 / 3} \mathrm{Ca}_{1 / 3} \mathrm{Mn}_{1-x} \mathrm{Al}_{x} \mathrm{O}_{3-\delta}$
}

\author{
J. Blasco, ${ }^{*}$ J. García, J. M. de Teresa, M. R. Ibarra, J. Perez, P. A. Algarabel, and C. Marquina \\ Departamento de Física de la Materia Condensada e Instituto de Ciencia de Materiales de Aragón, \\ Universidad de Zaragoza-Consejo Superior de Investigaciones Científicas, 50009-Zaragoza, Spain \\ C. Ritter \\ Institut Laue-Langevin, Boîte Postale 156, 38042 Grenoble Cédex, France
}

(Received 26 November 1996)

\begin{abstract}
The effect of the substitution for $\mathrm{Mn}$ with $\mathrm{Al}$ in the magnetoresistive perovskite $\mathrm{La}_{2 / 3} \mathrm{Ca}_{1 / 3} \mathrm{MnO}_{3}$ has been studied by preparing the series $\mathrm{La}_{2 / 3} \mathrm{Ca}_{1 / 3} \mathrm{Mn}_{1-x} \mathrm{Al}_{x} \mathrm{O}_{3}(x \leqslant 0.2)$. A careful study of the magnetic, structural, and transport properties has been carried out by means of electrical resistance, magnetoresistance, ac magnetic susceptibility, x-ray-diffraction, and neutron-diffraction techniques. Up to $x=0.05$ the Curie temperature (and the associated metal-insulator transition) decreases drastically with Al doping and the magnetoresistive properties do not change very much. For $x \geqslant 0.1$ the lattice spontaneously begins to lose oxygen atoms and for $x=0.2,3 \%$ of oxygen vacancies are present. This fact along with the random distribution of the $\mathrm{Al}$ atoms makes these compounds rather disordered from a structural and magnetic point of view. However, the magnetoresistance is enhanced, reaching colossal values of $10^{7} \%$ at $H=12 \mathrm{~T}$ at low temperatures for $x=0.2$. [S0163-1829(97)04414-7]
\end{abstract}

\section{INTRODUCTION}

The physical properties of the doped $\mathrm{LaMnO}_{3}$ samples is one of the most studied topics in the last years due to the observation of giant (and "colossal") magnetoresistance (GMR). ${ }^{1-4}$ The basic magnetic and structural properties of $R_{1-x} A_{x} \mathrm{MnO}_{3}$ ( $R$ : rare earth, $A: \mathrm{Ca}, \mathrm{Ba}, \mathrm{Sr}$ ) were widely studied in the past. ${ }^{5,6}$ Both end members are antiferromagnetic insulators, ${ }^{7}$ but doped compounds $(0.2 \leqslant x \leqslant 0.4)$ are ferromagnetic and metallic ${ }^{3}$ at low temperatures. This property was explained within the framework of the doubleexchange interaction by Zener ${ }^{8}$ but recently, it has been claimed that an additional mechanism such as a Jahn-Teller distortion may be required to explain the large magnetoresistance effects. ${ }^{9}$

Despite the exhaustive study of the effects of the rareearth replacement in these manganites (see, for instance, Refs. 10-12), very little is known about the influence of the substitution at Mn sites with other elements. The influence of $\mathrm{Fe}$ substitution ${ }^{13}$ on $\mathrm{Sm}$-based compounds and the effects of Al substitution on Pr-based compounds ${ }^{14}$ have recently been reported. We report here a thorough study on the effects of the replacement of $\mathrm{Mn}$ by $\mathrm{Al}$ in the archetypal giant magnetoresistive $\mathrm{La}_{2 / 3} \mathrm{Ca}_{1 / 3} \mathrm{MnO}_{3}$. The structural, magnetic, and electrical properties of this compound are well known. It undergoes a paraferromagnetic transition at $T_{c} \approx 260 \mathrm{~K}$. At this temperature an insulator-metal transition is observed. The highest magnetoresistance ratios are obtained around this temperature. ${ }^{15}$ In this compound two phenomena seem to be important: a charge-carrier localization process above $T_{c}$ (and suppressed at $T_{c}$ ), ${ }^{16,17}$ whose nature is still a matter of discussion, ${ }^{18}$ and the double-exchange interaction that causes the ferromagnetic ordering. The charge localization process above $T_{c}$ is characterized by an anomalous volume thermal expansion ${ }^{16,17}$ and the existence of short-range magnetic order far above $T_{c} .{ }^{17}$

A better understanding of the underlying physical mechanisms can be achieved by destabilizing the Mn sublattice. We have chosen $\mathrm{Al}$ to make the substitution because it has no magnetic moment and its atomic radius is smaller than the $\mathrm{Mn}$ one. Consequently, a rapid decrease in the intensity of the ferromagnetic interaction is expected and the localization process could also change. All this should be reflected in the magnetoresistance ratios. Moreover $\mathrm{LaAlO}_{3}$ is a well-known perovskite and consequently, $\mathrm{La}_{2 / 3} \mathrm{Ca}_{1 / 3} \mathrm{Mn}_{1-x} \mathrm{Al}_{x} \mathrm{O}_{3}$ solid solutions are expected in a wide range of concentration.

\section{EXPERIMENT}

$\mathrm{La}_{2 / 3} \mathrm{Ca}_{1 / 3} \mathrm{Mn}_{1-x} \mathrm{Al}_{x} \mathrm{O}_{3} \quad(x=0,0.01,0.03,0.05,0.1$, $0.15,0.2)$ samples were prepared by standard ceramic procedures. Stoichiometric proportions of $\mathrm{La}_{2} \mathrm{O}_{3}, \mathrm{CaCO}_{3}$, $\mathrm{Al}_{2} \mathrm{O}_{3}$, and $\mathrm{MnCO}_{3}$ with nominal purities not less than $99.99 \%$ were mixed and heated in air at $950{ }^{\circ} \mathrm{C}$ for $12 \mathrm{~h}$. After grinding they were pressed into bars and sintered in air at $1250^{\circ} \mathrm{C}$ for $48 \mathrm{~h}$ and $1400{ }^{\circ} \mathrm{C}$ for $12 \mathrm{~h}$ with intermediate grindings. One selected sample $(x=0.2)$ was also sintered in an oxygen current flow at $1000{ }^{\circ} \mathrm{C}$ and, at several temperatures $\left(800-1000{ }^{\circ} \mathrm{C}\right)$ under an oxygen pressure of 200 bars.

The oxygen content was analyzed using standard redox titration with $\mathrm{KMnO}_{4}$ and Mohr's salt. Step-scanned powder diffraction patterns were collected at room temperature using a D-Max Rigaku system with a rotating anode. The diffractometer was operated at $100 \mathrm{~mA}$ and $40 \mathrm{kV}$ with anode of $\mathrm{Cu}$ and a graphite monochromator was used to select the $\mathrm{Cu}$ $K \alpha_{1,2}$ radiation.

Magnetic measurements were carried out between 5 and $300 \mathrm{~K}$ by using a commercial Quantum Design superconducting quantum interference device magnetometer. The re- 
TABLE I. $\mathrm{Mn}^{4+}$ concentration and data obtained from x-ray-diffraction refinement at room temperature of lattice parameters $(\AA)$, unit-cell volume $\left(\AA^{3}\right)$, interatomic distances for $\mathrm{MnO}_{6}$ octahedron $(\AA)$, and angles between neighbors $\mathrm{MnO}_{6}$ octahedra (Deg.). O(1): apical oxygen; $\mathrm{O}(2)$ : basal oxygen.

\begin{tabular}{|c|c|c|c|c|c|c|c|c|}
\hline Sample & & $x=0$ & $x=0.01$ & $x=0.03$ & $x=0.05$ & $x=0.1$ & $x=0.15$ & $x=0.2$ \\
\hline & No. & & & & & & & \\
\hline $\mathrm{Mn}^{4+}(\%)$ & & 30 & 34 & 34 & 35 & 22 & 18 & 16 \\
\hline$a$ & & $5.4831(3)$ & $5.4699(2)$ & $5.4769(2)$ & $5.4643(3)$ & $5.4611(3)$ & $5.4524(3)$ & $5.4524(2)$ \\
\hline$b$ & & $5.4706(2)$ & $5.4592(2)$ & $5.4526(3)$ & $5.4533(2)$ & $5.4506(3)$ & $5.4438(2)$ & $5.4372(2)$ \\
\hline$c$ & & $7.7283(6)$ & $7.7068(8)$ & $7.6897(6)$ & $7.6999(7)$ & $7.6922(9)$ & $7.6749(6)$ & 7.6651(7) \\
\hline$V$ & & 231.82 & 230.13 & 229.64 & 229.44 & 228.96 & 227.80 & 227.17 \\
\hline $\mathrm{Mn}-\mathrm{O}(1)$ & 2 & $1.955(1)$ & $1.965(1)$ & $1.959(1)$ & $1.956(2)$ & $1.952(2)$ & $1.941(1)$ & $1.930(2)$ \\
\hline \multirow[t]{2}{*}{$\mathrm{Mn}-\mathrm{O}(2)$} & 2 & $1.963(6)$ & $1.973(7)$ & $2.001(7)$ & $1.974(6)$ & $1.959(7)$ & $1.967(4)$ & $1.953(6)$ \\
\hline & 2 & $1.959(6)$ & $1.945(6)$ & $1.919(5)$ & $1.934(5)$ & $1.956(5)$ & $1.950(3)$ & $1.960(6)$ \\
\hline$\langle\mathrm{Mn}-\mathrm{O}\rangle$ & & 1.959 & 1.961 & 1.960 & 1.955 & 1.956 & 1.953 & 1.948 \\
\hline \multicolumn{9}{|l|}{$\mathrm{Mn}-\mathrm{O}(1)-\mathrm{Mn}:$} \\
\hline & 2 & $160.9(3)$ & $157.3(3)$ & $157.6(2)$ & $159.4(3)$ & $157.8(4)$ & $154.4(4)$ & $157.7(4)$ \\
\hline \multicolumn{9}{|l|}{$\mathrm{Mn}-\mathrm{O}(2)-\mathrm{Mn}:$} \\
\hline & 4 & $160.4(8)$ & $160.7(3)$ & $160.4(6)$ & $161.7(7)$ & $161.4(6)$ & $163.7(5)$ & $163.2(7)$ \\
\hline$\langle\mathrm{Mn}-\mathrm{O}-\mathrm{Mn}\rangle$ & & 160.6 & 159.6 & 159.5 & 161.0 & 160.2 & 160.7 & 161.4 \\
\hline
\end{tabular}

sistance (magnetoresistance) measurements were performed by standard four-probe method. A superconducting coil was used to produce steady magnetic fields up to $12 \mathrm{~T}$ and the magnetic field was applied parallel to the current.

Neutron-diffraction measurements were performed at the high-flux reactor placed at the Institut Laue-Langevin. Two different instruments were used. For accurate refinement of the lattice parameters and the magnetic structure at low temperature, measurements were carried out on D2B, using a wavelength of $1.594 \AA$. For tracing the temperature dependence of the structural and magnetic properties, the instrument D1B was used. Using a wavelength of $2.52 \AA$, we studied the angular range $2.5^{\circ}-82.5^{\circ}$ at temperatures ranging from 1.5 to $320 \mathrm{~K}$.

\section{RESULTS AND DISCUSSION}

X-ray powder diffraction showed clean single-phase patterns for all samples. The diffractograms can be indexed in the Pbnm spatial group, very common in perovskites orthorhombically distorted and in agreement with related compounds. $^{2,3}$ Table I summarizes the relevant structural parameters obtained by Rietveld analysis of the diffractograms by using the FULLPROF program. ${ }^{19}$ The unit-cell volume and the average $\mathrm{Mn}-\mathrm{O}$ distance decrease with increasing the $\mathrm{Al}$ content. Both results are consistent with the lower size of $\mathrm{Al}^{3+}$. However, this substitution produces minor changes in the angles of the $\mathrm{MnO}_{6}$ octahedra, the average $\mathrm{Mn}-\mathrm{O}-\mathrm{Mn}$ angle remaining rather constant. Two effects can be expected by the partial replacement of $\mathrm{Mn}$ by $\mathrm{Al}$ in order to preserve the charge equilibrium: either an oxidation from $\mathrm{Mn}^{3+}$ to $\mathrm{Mn}^{4+}$ keeping the oxygen stoichiometry, or the formation of oxygen vacancies in the lattice. The first mechanism is preponderant at low $\mathrm{Al}$ concentrations as can be seen from the $\mathrm{Mn}^{4+}$ percentage in Table I. Therefore, samples with $x \leqslant 0.05$ are nominally stoichiometric. Nevertheless, the $\mathrm{Mn}^{4+}$ percentage decreases for higher $\mathrm{Al}$ content and consequently, oxygen vacancies are produced for $x \geqslant 0.1$. For in- stance, the actual formula for $x=0.2$ as measured by redox titration is $\mathrm{La}_{2 / 3} \mathrm{Ca}_{1 / 3} \mathrm{Mn}_{0.8} \mathrm{Al}_{0.2} \mathrm{O}_{2.9 \pm 0.02}$, which is in agreement with the formula $\mathrm{La}_{2 / 3} \mathrm{Ca}_{1 / 3} \mathrm{Mn}_{0.8} \mathrm{Al}_{0.2} \mathrm{O}_{2.93 \pm 0.02}$, obtained from Rietveld refinement of the neutron-diffraction data as can be seen below. One could think that this effect is not intrinsic but depending on the sample preparation. Several different methods of preparation have been used for the $x=0.2$ sample as has been described in the experimental section but in all of them, the value of $\mathrm{Mn}^{4+}$ obtained by titration redox was the same within the experimental error even for samples annealed at different temperatures under high oxygen pressure (200 bars).

Figure 1 shows the thermal dependence of the ac magnetic susceptibility for all samples. For low Al content (up to $x=0.05$ ) a rapid decrease in $T_{c}$ is observed but the curves are rather similar to the undoped compound one. It is noteworthy that the $T_{c}$ is not as well defined as for the undoped compound due to a certain degree of disorder in the Mn sublattice, responsible for the ferromagnetic interaction, caused by the Al presence. The same effect of $T_{c}$ broadening occurs in the Al-substituted Pr manganites. ${ }^{16}$ However, this effect does

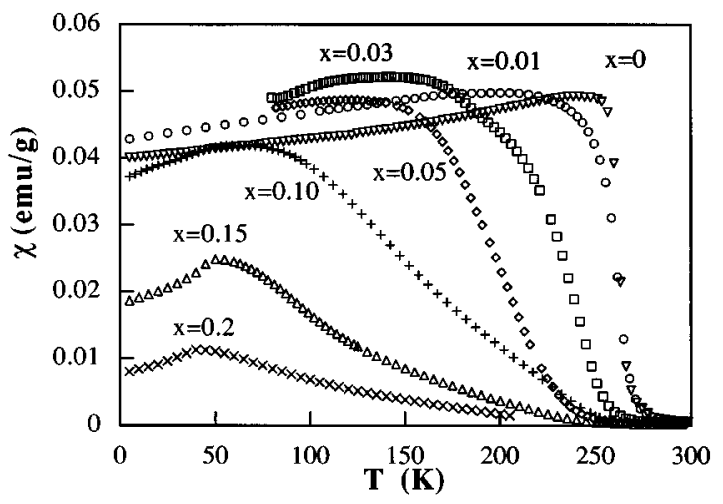

FIG. 1. ac magnetic susceptibility as a function of temperature for all studied compounds. 

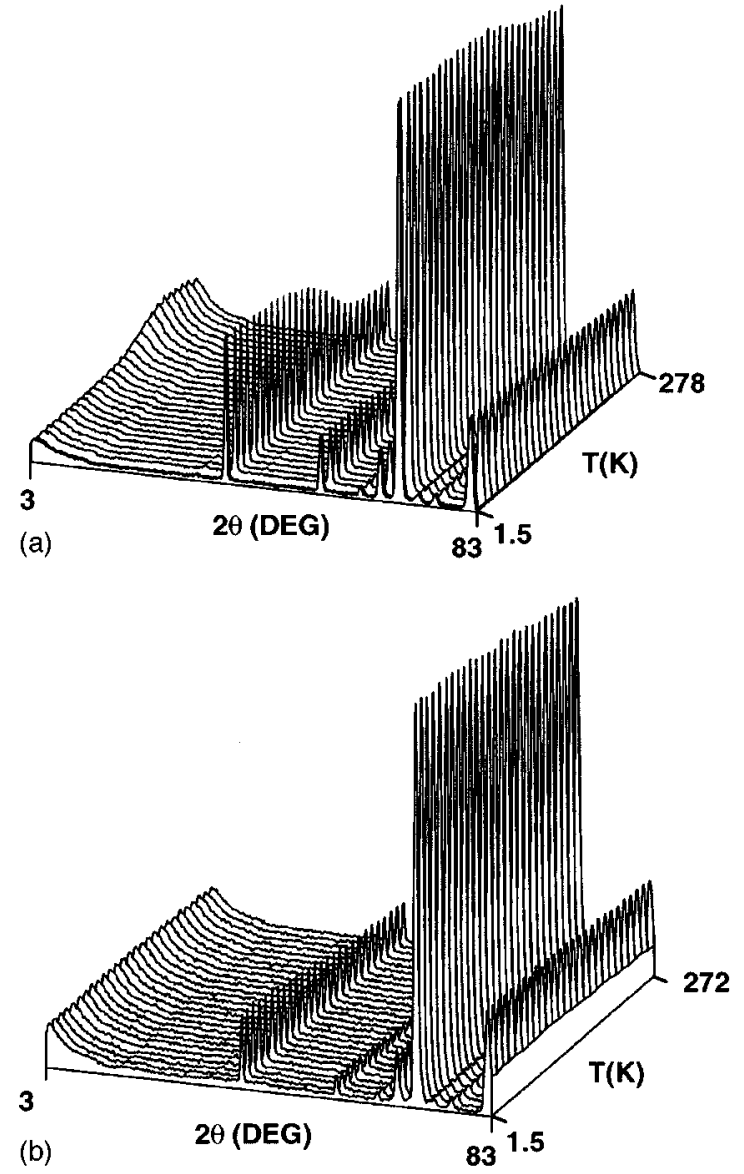

FIG. 2. Neutron-diffraction thermodiffractograms of the (a) $x=0.05$ and (b) $x=0.2$ compounds

not prevent the $\mathrm{Mn}$ ions from being all ferromagnetically ordered at low temperature. The refinement of the lowtemperature neutron-diffraction spectra shows that at $4 \mathrm{~K}$ the magnetic moment per Mn ion is $3.4 \mu_{B}$ for $x=0.05$, which is close to the theoretical saturation magnetic moment of 3.6 $\mu_{B}$. This is important because it indicates that at low temperatures the samples with $x \leqslant 0.05$ are magnetically homogeneous. The magnetic behavior of the compounds with $x \geqslant 0.1$ is completely different. From the ac magnetic susceptibility, $T_{c}$ cannot be straightforwardly obtained as the curves are rather smooth and a cusp is developed at around $60 \mathrm{~K}$ that is more pronounced for greater $\mathrm{Al}$ content. From the susceptibility measurements alone, it is not possible to draw trusty conclusions and consequently, a microscopic study has been carried out by measuring neutron diffraction.

The thermodiffractograms at scattering angles between $3^{\circ}$ and $83^{\circ}$ and at temperatures ranging from 1.5 to $320 \mathrm{~K}$ are shown in Fig. 2 for the compounds with $x=0.05$ and 0.2. For $x=0.05$ the ferromagnetic ordering is clearly seen through the increase in intensity of the first Bragg nuclear peak at $T_{c} \approx 200 \mathrm{~K}$, in agreement with the ac susceptibility measurements. Another feature reminiscent of the $x=0 \mathrm{com}-$ pound is the observation of small-angle neutron scattering (SANS). In $\mathrm{La}_{2 / 3} \mathrm{Ca}_{1 / 3} \mathrm{MnO}_{3}$ it was shown that as $T_{c}$ is approached from above, ferromagnetic clusters develop bringing about a strong contribution to the small-angle scattering. ${ }^{17}$ One can see that the same effect takes place for

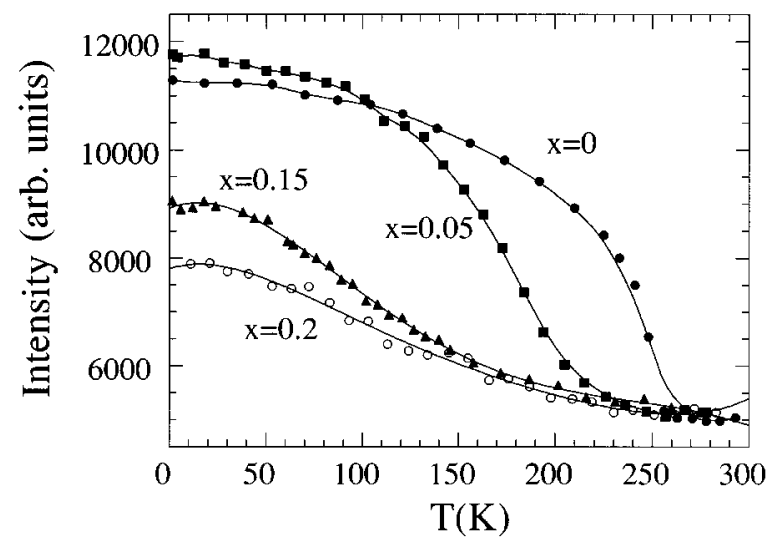

FIG. 3. Temperature dependence of the intensity of the Bragg peak $\left(\begin{array}{lll}1 & 1 & 0\end{array}\right)$ for $x=0,0.05,0.15$, and 0.2 . The rise corresponds to the onset of the magnetic contribution. Lines are visual guides.

the $x=0.05$ compound. A clear SANS contribution appears above $T_{c}$ that is suppressed at $T_{c}$, when the paraferromagnetic and insulator-metal transitions occur.

In Fig. 2(b) the thermodiffractogram for $x=0.2$ indicates that the characteristics of a unique transition temperature are not present. The intensity of the first Bragg nuclear peak, (1 10 ), as a function of temperature is plotted in Fig. 3. A magnetic contribution appears at $200 \mathrm{~K}$ and rises steadily down to low temperatures. Instead of having a typical ferromagnetic dependence on temperature as it occurs in $x=0.05$, for $x=0.2$ (and $x=0.15$ ) the magnetic intensity behaves in a rather unusual way. The Rietveld refinement at 4 $\mathrm{K}$ indicates that the magnetic moment per Mn ion (see Table II) is $2.5 \mu_{B}$ for $x=0.15$ and $1.85 \mu_{B}$ for $x=0.2$, far from the saturation magnetic moment. These results are in agreement with the ac susceptibility measurements and can be explained under the assumption that the compounds are magnetically inhomogeneous. This magnetic disorder has two sources: the presence of $\mathrm{Al}$ in the $\mathrm{Mn}$ sublattice and the spontaneous appearance of oxygen vacancies. The distribution of both of them in the lattice is plausible to be random in a first approximation. Statically some regions of the sample will be more populated with them than the rest. If one thinks in the $x=0.2$ compound, with a $20 \%$ of $\mathrm{Al}$ impurities and $3 \%$ of oxygen vacancies, the sample will be possibly very disordered from a structural point of view. This structural disorder must lead to high magnetic inhomogeneity. The ferromagnetic double-exchange mechanism is a process consisting of a Mn-Mn indirect interaction through the oxygen orbitals. Consequently, it will be very sensitive to the presence of $\mathrm{Al}$ impurities and oxygen vacancies. In regions of high concentration of them, the double-exchange will be thwarted. The scenario that comes up from the neutron measurements for $x \geqslant 0.1$ is one where there is a continuous distribution of Curie temperatures from $200 \mathrm{~K}$ down and probably at the lowest temperature some regions are clusterlike spin-glass rather than ferromagnetic. Otherwise, the refined magnetic moment per Mn ion would not be so far from the expected value for saturation. It should be mentioned that regions with magnetic coherent length of the order of $10^{3} \AA$ will contribute in intensity to the magnetic scattering on Bragg peaks. Consequently, regions of much smaller magnetic coherent length will not give such contribution and should be consid- 
TABLE II. Data at $1.5 \mathrm{~K}$ obtained from neutron refinement of lattice parameters $(\AA)$, unit-cell volume $\left(\AA^{3}\right)$, interatomic distances for $\mathrm{MnO}_{6}$ octahedron $(\AA)$, angles between neighbors $\mathrm{MnO}_{6}$ octahedra (Deg.), and ferromagnetic moment $\left(\mu_{B} / \mathrm{Mn}\right)$.

\begin{tabular}{lcccc}
\hline \hline Sample & & $x=0.05$ & $x=0.15$ & $x=0.2$ \\
\hline & No. & & & \\
$a$ & & $5.4572(5)$ & $5.4538(4)$ & $5.4488(9)$ \\
$b$ & & $5.4428(4)$ & $5.4368(4)$ & $5.4308(8)$ \\
$c$ & & $7.6876(6)$ & $7.6741(6)$ & $7.6580(12)$ \\
$V$ & & 228.34 & 227.55 & 226.61 \\
$\mathrm{Mn}-\mathrm{O}(1):$ & 2 & $1.956(1)$ & $1.951(1)$ & $1.948(1)$ \\
$\mathrm{Mn}-\mathrm{O}(2):$ & 2 & $1.949(3)$ & $1.918(4)$ & $1.907(6)$ \\
& 2 & $1.952(3)$ & $1.974(4)$ & $1.980(6)$ \\
$\langle\mathrm{Mn}-\mathrm{O}\rangle$ & & 1.952 & 1.948 & 1.945 \\
$\mathrm{Mn}-\mathrm{O}(1)-\mathrm{Mn}:$ & 2 & $169.3(1)$ & $169.4(1)$ & $169.4(2)$ \\
$\mathrm{Mn}-\mathrm{O}(2)-\mathrm{Mn}:$ & 4 & $171.1(1)$ & $171.5(1)$ & $171.6(1)$ \\
$\langle\mathrm{Mn}-\mathrm{O}-\mathrm{Mn}\rangle$ & & 170.5 & 170.8 & 170.85 \\
$\mu$ & & 3.41 & 2.5 & 1.85 \\
\hline \hline
\end{tabular}

ered cluster-glass-type instead of ferromagnetic. In Fig. 4 the Rietveld refinement of the $x=0.15$ compound at $1.5 \mathrm{~K}$ is shown. Some parameters obtained from the fit for this compound and for the other compounds are listed in Table II.

The other feature of carrier localization above $T_{c}$ in $\mathrm{La}_{2 / 3} \mathrm{Ca}_{1 / 3} \mathrm{MnO}_{3}$ is the existence of an anomalous volume thermal expansion with a jump at $T_{c} \cdot{ }^{16,17}$ In Fig. 5 the volume thermal expansion calculated with the lattice parameters obtained from the neutron-diffraction spectra fits is shown. A volume anomaly is detected at around $T_{c}$ for $x=0.05$ while it

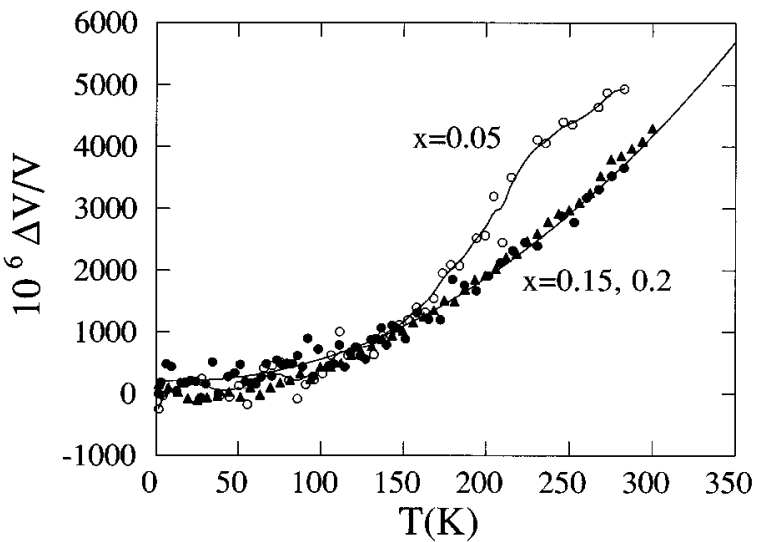

FIG. 5. Volume thermal expansion calculated from the thermal dependence of the lattice parameters as obtained from the fits of the spectra to the $\mathrm{Pbnm}$ space group. Closed dots and triangles correspond, respectively, to $x=0.15$ and $x=0.2$. Open circles correspond to $x=0.05$. Lines are visual guides.

is no longer visible for $x \geqslant 0.1$ because there is not a magnetic transition at a specific temperature. Correspondingly, the characteristic SANS intensity above $T_{c}$ has also disappeared [see Fig. 2(b)]. Although D1B is not a high-resolution spectrometer for lattice parameters and the absolute values should be taken with care, qualitatively one can see the anomalous volume thermal expansion for $x=0.05$.

The resistivity as a function of temperature is plotted in Fig. 6. The results nicely reflect the microscopic behavior but under no circumstances should one draw microscopic conclusions from the resistivity measurements alone. Apparently

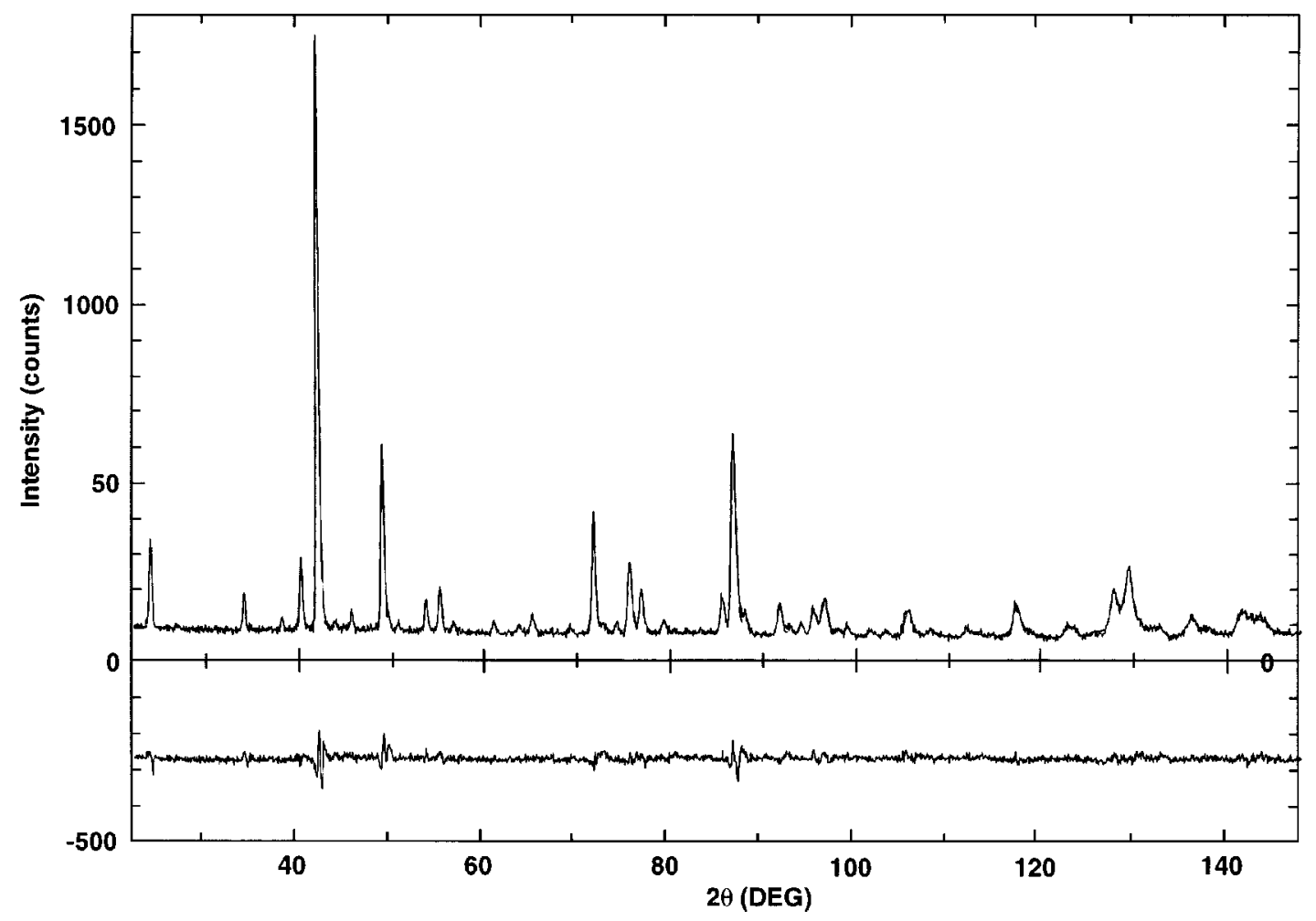

FIG. 4. Rietveld refinement of the spectra at $T=1.5 \mathrm{~K}$ of the $x=0.15$ compound. The main parameters obtained from this fit are listed in Table II. The difference between measured and calculated data is plotted below. 


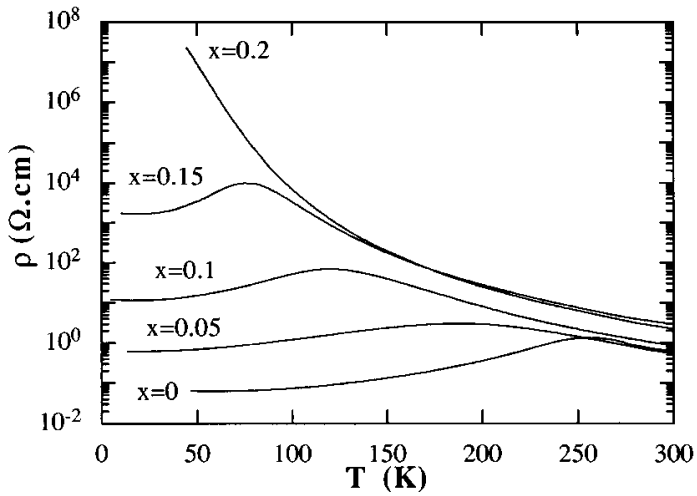

FIG. 6. Resistivity as a function of temperature for the $x=0$, $0.05,0.1,0.15$, and 0.2 compounds.

the curves are quite similar for compounds with $x \leqslant 0.15$, with a maximum that shifts to lower temperatures as the $\mathrm{Al}$ content increases. However, as we have shown with the neutron measurements, the microscopic behavior is different. For $x=0$, it is well known that at the paraferromagnetic transition temperature, $T_{c}$, a metal-insulator transition occurs simultaneously. The maximum of the curve for $x=0.05$ should be interpreted in the same way because all the hallmarks of this transition are present: volume anomaly at $T_{c}$ and SANS above $T_{c}$. In this compound the maximum is rather smooth because of the $T_{c}$ broadening aforementioned. For the $x=0.1$ and $x=0.15$ compounds the maximum does not correspond to a true insulator-metal transition. The maximum occurs at a lower temperature than the onset of longrange magnetic order. In these compounds where the electrical and magnetic behavior is so tight, the magnetic inhomogeneity should alter the transport properties. As the ferromagnetic state should give metallic behavior and the paramagnetic (or spin-glass) state should give insulator behavior, the presence of magnetically inhomogeneous regions must bring about the coexistence of conductive and insulating regions within the sample. The important parameters are the local magnetic coherence length and the free mean path for the electron scattering in a spin-aligned region. If the electron moves in a region of magnetic coherence length smaller than this electronic free mean path, it will be additionally scattered. The macroscopic behavior will be a balance that depends on the extent of the conductive and insulating regions. Due to this, for $x=0.2$ a maximum is no longer seen and the sample remains insulator up to $50 \mathrm{~K}$, where the resistivity is so high that it saturates our experimental setup.

The magnetoresistance curves for some selected samples are shown in Fig. 7. The magnetoresistance is defined here as $\operatorname{MR}(\%)=100 \times|R(H=12 \mathrm{~T})-R(H=0)| / R(H=12 \mathrm{~T})$. It is observed that as the $\mathrm{Al}$ content increases, the MR ratio increases, but at lower temperatures, which is not suitable for technological applications at room temperature. For $x=0.2$ the MR ratios are very high and the effect should be ascribed to the effect of the magnetic field on the insulating regions. It was shown in $\left(\mathrm{La}_{2 / 3} \mathrm{~Tb}_{1 / 3}\right)_{2 / 3} \mathrm{Ca}_{1 / 3} \mathrm{MnO}_{3},{ }^{12,20}$ which is cluster glass at low temperatures, that the magnetic field can change the resistivity in 11 orders of magnitude. In fact, it is like an insulator-metal transition. In the metallic regions the resis-

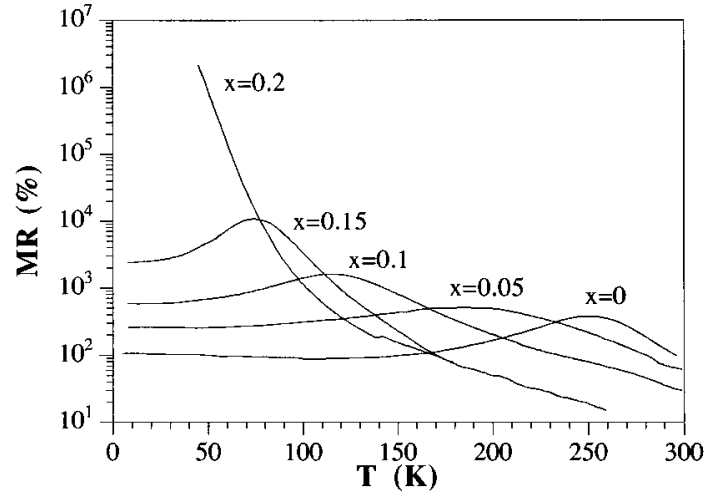

FIG. 7. Magnetoresistance at $H=12 \mathrm{~T}$ (see text) as a function of temperature for the $x=0,0.05,0.1,0.15$, and 0.2 compounds.

tance comes from the misalignment of spins. The effect of the magnetic field is to align them and the expected magnetoresistance is much smaller. In Fig. 8(a) the resistivity for $x=0.2$ at 0 and $12 \mathrm{~T}$ is shown. As can be seen, the residual resistivity at $12 \mathrm{~T}$ remains very high for $x=0.2$ sample. This was also observed in $\left(\mathrm{La}_{2 / 3} \mathrm{~Tb}_{1 / 3}\right)_{2 / 3} \mathrm{Ca}_{1 / 3} \mathrm{MnO}_{3},{ }^{12,20}$ due to the nature of the cluster-glass state. We have also measured ac magnetic susceptibility at different frequencies to check if the spin-glass regions present in the $x=0.2$ compound bring about a shift in the maximum. The results shown in Fig. 8(b) confirm this hypothesis.

The macroscopic results obtained for the $\mathrm{La}_{2 / 3} \mathrm{Ca}_{1 / 3} \mathrm{Mn}_{1-x} \mathrm{Al}_{x} \mathrm{O}_{3-\delta}$ series are very similar to those obtained for the $\left(\mathrm{La}_{1-x} \mathrm{~Tb}_{x}\right)_{2 / 3} \mathrm{Ca}_{1 / 3} \mathrm{MnO}_{3}$, (Ref. 12) and
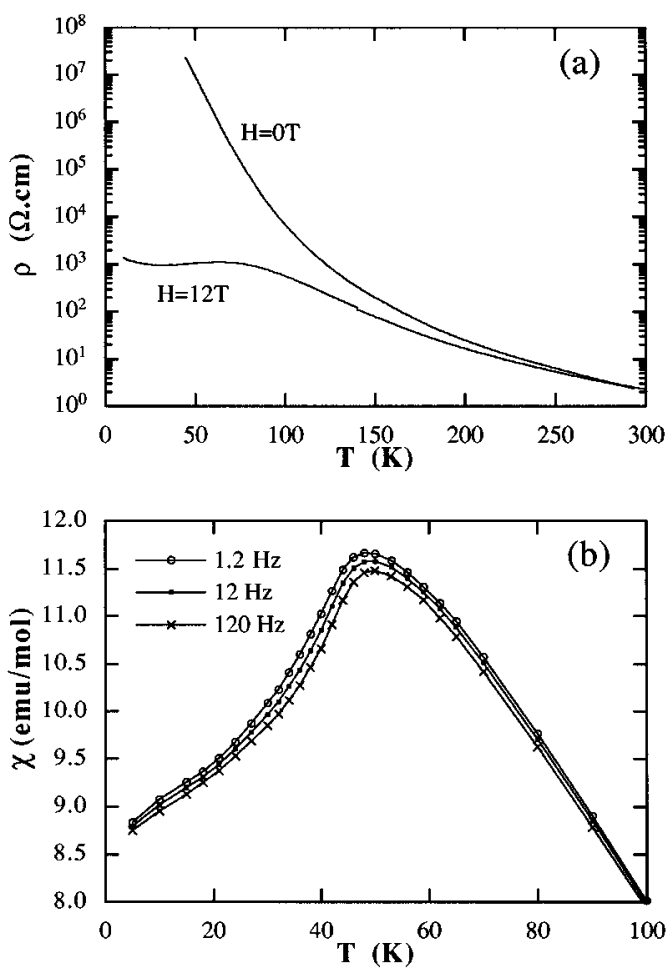

FIG. 8. (a) Resistivity without and under magnetic field (12 T) for the $x=0.2$ compound. (b) Frequency dependence of the cusp seen in ac susceptibility for the $x=0.2$ compound. 
other related series ${ }^{21}$ that exhibit giant magnetoresistance: when the double-exchange interaction is weakened, the $T_{c}$ diminishes and the magnetoresistance ratios are enhanced but at lower temperatures, which is a drawback for technological applications. However, the microscopic mechanisms in both kinds of systems are different. When the substitution takes place in the rare-earth site with a smaller ion, the Mn$\mathrm{O}-\mathrm{Mn}$ angle decreases, disfavoring the transfer integral between Mn ions and consequently weakening the doubleexchange ferromagnetic mechanism. In those compounds the disorder that occurs in the rare-earth sites is a second-order correction on the magnetic homogeneity because the magnetism is governed by the $\mathrm{Mn}$ and $\mathrm{O}$ ions positions. On the other hand, when the Mn sublattice is destabilized by substituting $\mathrm{Mn}$ by nonmagnetic ions like $\mathrm{Al}$, the compounds result in inhomogeneous magnetic systems. In the studied $\mathrm{La}_{2 / 3} \mathrm{Ca}_{1 / 3} \mathrm{Mn}_{1-x} \mathrm{Al}_{x} \mathrm{O}_{3-\delta}$ compounds, this disorder is enhanced by the presence of oxygen vacancies and at low temperatures there is coexistence of regions in the samples with very different magnetic coherence lengths. In this series we have found that for high $\mathrm{Al}$ contents the magnetoresistance appears not around $T_{c}$ (which is not well defined any more) but at low temperatures due to the effect of the magnetic field on the insulating regions (those with short magnetic coherence length).

\section{CONCLUSIONS}

By replacing $\mathrm{Mn}$ by $\mathrm{Al}$ in the giant magnetoresistive perovskite $\mathrm{La}_{2 / 3} \mathrm{Ca}_{1 / 3} \mathrm{MnO}_{3}$, colossal magnetoresistance ratios appear at low temperture for high Al contents $(x=0.2)$. For $x \leqslant 0.05$, the properties of the undoped compound are retained except for a broadening of the Curie temperature. For $x \geqslant 0.1$, the compounds become magnetically inhomogeneous as a result of the structural disorder caused in the $\mathrm{Mn}$ sublattice and the unexpected presence of intrinsic oxygen vacancies. Neutron-diffraction measurements show that a single $T_{c}$ does not exist but regions of very different magnetic coherence length. This brings about the coexistence of metallic and insulating regions at low temperature, the magnetoresistance being caused mainly by the effect of the magnetic field on the insulating regions. Once more the electrical properties of the manganese perovskites have been found to be a consequence of the microscopic structural and magnetic behavior.

\section{ACKNOWLEDGMENT}

The Spanish authors are grateful to the Spanish DGICYT for financial support under Project Nos. MAT 96-0491, MAT96-826, and APC95-123.

${ }^{9}$ A. J. Millis, P. B. Littlewood, and B. I. Shraiman, Phys. Rev. Lett. 74, 5144 (1995).

${ }^{10}$ H. Y. Hwang et al., Phys. Rev. Lett. 75, 914 (1995).

${ }^{11}$ V. Caignaert et al., Solid State Commun. 95, 357 (1995).

${ }^{12}$ J. Blasco et al., J. Phys. Condens. Matter 8, 7427 (1996).

${ }^{13}$ F. Damay et al., J. Solid State Commun. 124, 385 (1996).

${ }^{14}$ C. Martin, A. Maignan, and B. Raveau, J. Mater. Chem. 6, 1245 (1996).

${ }^{15}$ P. Schiffer et al., Phys. Rev. Lett. 75, 3336 (1995).

${ }^{16}$ M. R. Ibarra et al., Phys. Rev. Lett. 75, 3541 (1995).

${ }^{17}$ J. M. De Teresa et al., Phys. Rev. B 54, 1187 (1996).

${ }^{18}$ J. M. De Teresa et al., Phys. Rev. B 54, R12 689 (1996).

${ }^{19}$ J. Rodriguez-Carvajal, Physica B 192, 55 (1992).

${ }^{20}$ J. M. De Teresa et al., Phys. Rev. Lett. 76, 3392 (1996).

${ }^{21}$ J. Fontcuberta et al., Phys. Rev. Lett. 76, 1122 (1996). 